\title{
Microemulsions: Breakthrough Electrolytes for Redox Flow Batteries
}

\author{
Brian A. Barth ${ }^{1}$, Adam Imel ${ }^{1}$, K. McKensie Nelms ${ }^{1}$, Gabriel A. Goenaga ${ }^{1}$ and \\ Thomas Zawodzinski ${ }^{1,2 *}$ \\ ${ }^{1}$ Department of Chemical and Biomolecular Engineering, University of Tennessee, Knoxville, TN, United States, ${ }^{2}$ Oak Ridge \\ National Laboratory, Oak Ridge, TN, United States
}

Aqueous and non-aqueous redox flow batteries (RFBs) have limited energy and current densities, respectively, due to the nature of the electrolytes. New approaches to electrolyte design are needed to improve the performance of RFBs. In this work, we combined a highly conductive aqueous phase and an organic redox-active phase in a microemulsion to formulate a novel RFB electrolyte. As a proof-of-concept, we demonstrate an RFB using this microemulsion electrolyte with maximum current density of $17.5 \mathrm{~mA} \cdot \mathrm{cm}^{-2}$ with a $0.19 \mathrm{M}$ posolyte and $0.09 \mathrm{M}$ negolyte at a flow rate of only $\sim 2.5 \mathrm{ml} \cdot \mathrm{min}^{-1}$, comparable to early vanadium electrolyte RFBs at similar flow rates on a per molar basis. The novel active

OPEN ACCESS

Edited by:

Jennifer L. Schaefer, University of Notre Dame,

United States

Reviewed by:

Fikile Brushett,

Massachusetts Institute of Technology, United States

Tianbiao Liu,

Utah State University, United States

Yu Zhao,

Soochow University, China

*Correspondence:

Thomas Zawodzinski tzawodzi@utk.edu

Specialty section: This article was submitted to Electrochemistry, a section of the journal Frontiers in Chemistry

Received: 08 December 2021 Accepted: 31 January 2022

Published: 03 March 2022

Citation:

Barth BA, Imel A, Nelms KM, Goenaga GA and Zawodzinski T (2022) Microemulsions: Breakthrough Electrolytes for Redox Flow Batteries.

Front. Chem. 10:831200.

doi: $10.3389 /$ fchem.2022.831200 negolyte component is an inexpensive oil-soluble vitamin $\left(\mathrm{K}_{3}\right)$. By combining aqueous and organic phases, the solvent potential window and energy density may be increased without sacrificing current density and new redox couples may be accessed. Microemulsion electrolytes show great promise for improved performance and increased energy densities in aqueous RFBs but the path forward is complex. We end with discussion of areas that need work to achieve the potential of these electrolytes.

Keywords: microemulsions, redox flow batteries, electrolytes, energy storage, electrochemical devices

\section{INTRODUCTION}

Redox flow batteries (RFBs) are a promising energy storage technology. However, both aqueous and non-aqueous RFBs suffer from limitations imposed by electrolyte choice (Luo et al., 2019a). Nonaqueous electrolytes often have low redox species concentrations and poor conductivity, which limits current density (Sun et al., 2017). Aqueous electrolytes have limited energy densities due to the narrow potential window of water, and suitable redox materials are restricted to metal ions or substituted organic redox active species. While progress has been made in the design and implementation of aqueous organic RFBs (Hu et al., 2017; Luo et al., 2019b), an alternative approach to is combine redox-containing oil phases with aqueous electrolyte phases in the same solution.

As part of our work on 'Breakthrough Electrolytes for Energy Storage', we adopt this path to broadening the possibilities for RFBs. Microemulsions provide a novel electrolyte design that may overcome existing limitations by providing independent pathways for different functions-ion conduction and redox reaction-allowing us to carry out reactions on redox couples that are not water soluble with the benefit of solution conductivity characteristic of an aqueous system. Microemulsions are spontaneously forming, thermodynamically stable mixtures of oil, water, and emulsifiers, with complex and dynamic nanometer-scale structures. These structures can vary greatly from discrete droplets to bicontinuous networks, where both oil and water are continuous over longer length-scales. There is an expansive history of microemulsion media 
used in electroanalytical and electro-synthetic studies (Mackay 1994; Rusling 1994; Qutubuddin 1999), but microemulsions have only recently been proposed as RFB electrolytes (Peng et al., 2020; Shen et al., 2021; Peng et al., 2021a; Peng et al., 2021b). We note explicitly that the presence of an electroactive component in the oil phase is the critical aspect of this work, providing access to not only solubility but likely additional reactivity patterns. We also note that the electrochemical conversion may result in the transfer of the oxidized or reduced species from oil to water phase (vide infra) (Mackay 1994), analogous to probe partitioning in aqueous micelle solutions (Oshawa et al., 1980). Nonetheless, we suggest that the microemulsion electrolyte must have one or more active species in the oilphase if the system is to have meaning outside that of a simple surfactant-containing electrochemical system. This study is intended as a starting point for more extensive work-a proof of concept. Accordingly, we did not seek to optimize conditions or materials but rather simply used what we have used in more fundamental studies. We used methods that we have previously used and, indeed, help standardize in the context of flow batteries. Since we are embarking on a rather different direction for flow batteries, we necessarily build primarily on our recent publications on electrochemistry and structure in microemulsions, but we also want to clearly acknowledge the extensive work in the past.

Electroanalytical microemulsion studies have commonly chosen ferrocene as a model redox species (Iwunze et al., 1990; Mackay et al., 1990; Jha et al., 1995; Mackay et al., 1996; Zu and Rusling 1997; Mo and Li 2007) due to its reversible, outersphere electron transfer character (McCreery 2008). We used ferrocene and polysorbate $\left(\right.$ TWEEN $^{\circledR}$ ) 20, a non-ionic surfactant known to be relatively insensitive to solution ionic strength, to probe microemulsion structure-electrochemical behavior relationships (Peng et al., 2020). Unlike previous studies where redox material was added at low concentrations to function as a probe (Iwunze et al., 1990; Mackay et al., 1990; Jha et al., 1995; Mackay et al., 1996; Zu and Rusling 1997; Mo and Li 2007), we suggested that microemulsions could be suitable RFB electrolytes if redox species concentration was increased (Peng et al., 2020). Using microemulsions, it is possible to increase energy density with respect to aqueous RFBs by widening the potential window through surfactant-electrode interactions (Peng et al., 2020; Hou et al., 2017), or the volumetric capacity, through use of organic redox species which undergo multi-electron transfer reactions. It is also possible to increase the achievable current density, relative to non-aqueous RFBs, by increasing the solution conductivity and the concentration of redox active material. The intimate mixing of oil, water, and emulsifier in microemulsion solutions provides a substantial benefit: the choice of organic solvent is no longer constrained by supporting electrolyte solubility because solution conductivity is maintained through the aqueous phase. Therefore, organic solvents and redox species may be chosen with the intent of maximizing redox species solubility.

The ferrocene-polysorbate 20 microemulsion system (Peng et al., 2020) does not have as large of a volumetric capacity or as positive of a redox potential as the more mature vanadium RFB posolyte. However, as a model system it is a viable posolyte for early development of a microemulsion RFB, given its well-documented structure and electrochemical behavior (Peng et al., 2020; Shen et al., 2021). We chose menadione, a vitamin quinone (vitamin $\mathrm{K}_{3}$ ), as a suitable negolyte because of its low cost, lack of toxicity, solubility in toluene, and the ability to undergo two electron transfer reactions. Hence, sacrifices in volumetric capacity originating from solubilizing redox material in only one phase of a multi-phase solution can be partly mitigated. Here we present the first microemulsion electrolyte RFB, identify the strengths and weaknesses of using microemulsion electrolytes, and provide a path for future implementation of this technology.

\section{METHODS}

\section{Microemulsion Preparation}

Polysorbate 20 (Acros, >95\%), sodium dodecyl sulfate (SDS; Fisher, $\geq 99 \%$ ), 1-butanol (Fisher, 99.98\%), toluene (Fisher, 99.9\%), potassium nitrate (Fisher, $\leq 3 \mathrm{ppm}$ heavy metals, iron), ferrocene (Alfa Aesar, 99\%), and menadione (MP Biomedicals, $\geq$ $98 \%)$ were used as received. Chemical structures of surfactants and electroactive species are shown in Figure $\mathbf{1 .}$

\section{Polysorbate 20 Microemulsions}

Emulsifier stock solution was prepared using a 4.7:1 mass ratio solution of polysorbate 20:1-butanol. Electroactive material (ferrocene or menadione) was solubilized in toluene, and then combined with the emulsifier stock solution and $\mathrm{KNO}_{3}(\mathrm{aq})$ to reach the desired composition. The resulting mixture was shaken by hand to produce a clear, single-phase microemulsion.

\section{Sodium Dodecyl Sulfate Microemulsions}

An aqueous emulsifier stock solution was prepared by combining $0.5 m \mathrm{KNO}_{3}(\mathrm{aq})$, SDS, and 1-butanol in a mass ratio 75/12.5/12.5 (0.5m $\mathrm{KNO}_{3} / \mathrm{SDS} / 1$-butanol). Electroactive material (ferrocene or menadione) was solubilized in toluene, and then combined with the aqueous emulsifier stock solution to reach desired composition. The mixture was shaken by hand to produce a clear, single-phase microemulsion.

\section{Nuclear Magnetic Resonance Spectroscopy}

Microemulsion bulk morphology (oil droplets, water droplets, or bicontinuous) was determined from oil and water diffusivities obtained by stimulated pulse echo NMR using a Bruker $400 \mathrm{MHz}$ Ascend spectrometer. Samples were prepared as described above, with the exception that the aqueous salt solution was $10 \% \mathrm{D}_{2} \mathrm{O}$ (for signal locking). The applied pulse sequence was $\Delta=50 \mathrm{~ms}, \delta=2 \mathrm{~ms}$, and g varied linearly from $0.963 \mathrm{G} / \mathrm{cm}$ to $45.743 \mathrm{G} / \mathrm{cm}$ (for microemulsions without redox species present) or $47.187 \mathrm{G} / \mathrm{cm}$ (for microemulsions with redox species present). Diffusivities were calculated by fitting the decaying signal areas to the Stejskal-Tanner equation (Stejskal and Tanner 1965): 
<smiles>CCCCCCCCCCCCOP(=O)(O)O[NH3+]</smiles>

sodium dodecyl sulfate

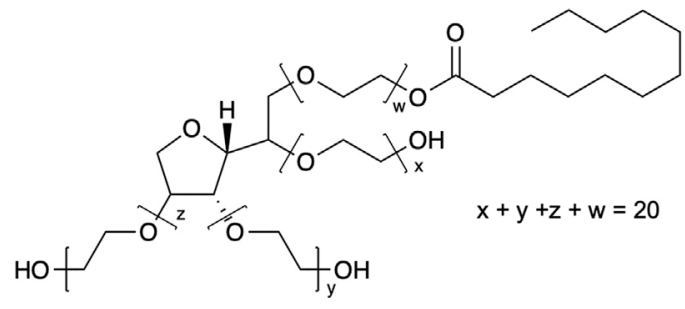

polysorbate 20

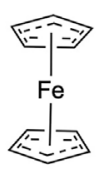

ferrocene<smiles>CC1=CC(=O)c2ccccc2C1=O</smiles>

menadione

FIGURE 1 | Chemical structures of surfactants and electroactive species.

$$
\frac{I}{I_{0}}=e^{-D \gamma^{2} g^{2} \delta^{2}(\Delta-\delta / 3)}
$$

where $I / I_{0}$ is ratio of signal intensity to maximum signal intensity (in the absence of an applied $\mathrm{z}$-gradient), $\mathrm{D}\left(\mathrm{cm}^{2} / \mathrm{s}\right)$ is the diffusivity, $\gamma(\mathrm{rad} /(\mathrm{s} \cdot \mathrm{G}))$ is the gyromagnetic ratio of the nucleus, $g(\mathrm{G} / \mathrm{cm})$ is the gradient strength, $\delta(\mathrm{s})$ is pulse time, and $\Delta(\mathrm{s})$ is the diffusion time.

\section{Cyclic Voltammetry}

Cyclic voltammetry measurements were performed using a BioLogic VMP3 potentiostat and EC-lab software. Approximately $10 \mathrm{ml}$ of microemulsion solution was added to a clean electrochemical cell fitted with a Teflon cap. A glassy carbon working electrode was polished using 5- and 0.05micron alumina powder slurries on polishing pads prior to use. A glassy carbon working electrode, a saturated calomel reference electrode, and a Pt wire counter electrode were inserted into the solution through the fitted cap. The solution was purged with nitrogen for $10 \mathrm{~min}$ prior to data collection and a nitrogen blanket was maintained, without agitating the solution, during data acquisition. Solution resistance was approximated as the high frequency resistance (HFR) obtained by potentiostatic electrochemical impedance spectroscopy (PEIS). Cyclic voltammograms were recorded with iR-compensation using the previously determined solution resistance values.

\section{Conductivity}

Conductivity measurements were performed using an in-house conductivity cell (Figure 2) operated by a BioLogic SP-200 potentiostat and EC-lab software Potentiostatic electrochemical impedance spectroscopy was performed by applying $0 \mathrm{~V}$ DC bias, with a $10 \mathrm{mV}$ AC amplitude perturbation while scanning the frequencies from 200 to
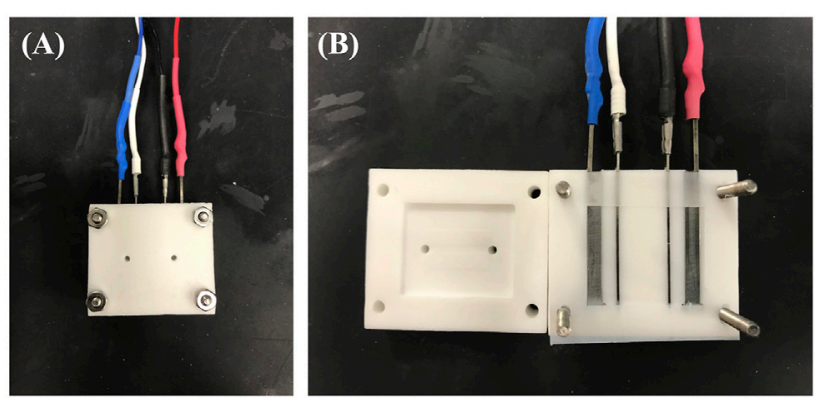

FIGURE 2 | Conductivity cell from (A) closed and (B) open viewpoint. Outer terminals (blue and red) are power leads and inner terminals (white and black) are sensing leads.

$1 \mathrm{kHz}$. The solution resistance was obtained from the HFR intercept determined from the Nyquist plot. Solution conductivity was calculated using

$$
\sigma\left(S \cdot \mathrm{cm}^{-1}\right)=\frac{1}{\mathrm{HFR}} \cdot \frac{\text { length }}{\text { cross }- \text { sectional area }}
$$

where the length and cross-sectional area are determined by the cell geometry. The methodology and cell were validated by comparison with a conductivity standard solution (Mettler Toledo, $0.00056 \mathrm{M} \mathrm{KCl}$ ), where the measured conductivity was within $2.4 \%$ of the standard value.

\section{RFB Testing}

We note at the outset that this study is intended as a starting point for more extensive work-a proof of concept. Accordingly, we did not seek to optimize conditions or materials but rather simply used what we have used in more fundamental studies. We used methods that we have previously used and, indeed, help standardize in the context of flow batteries. 


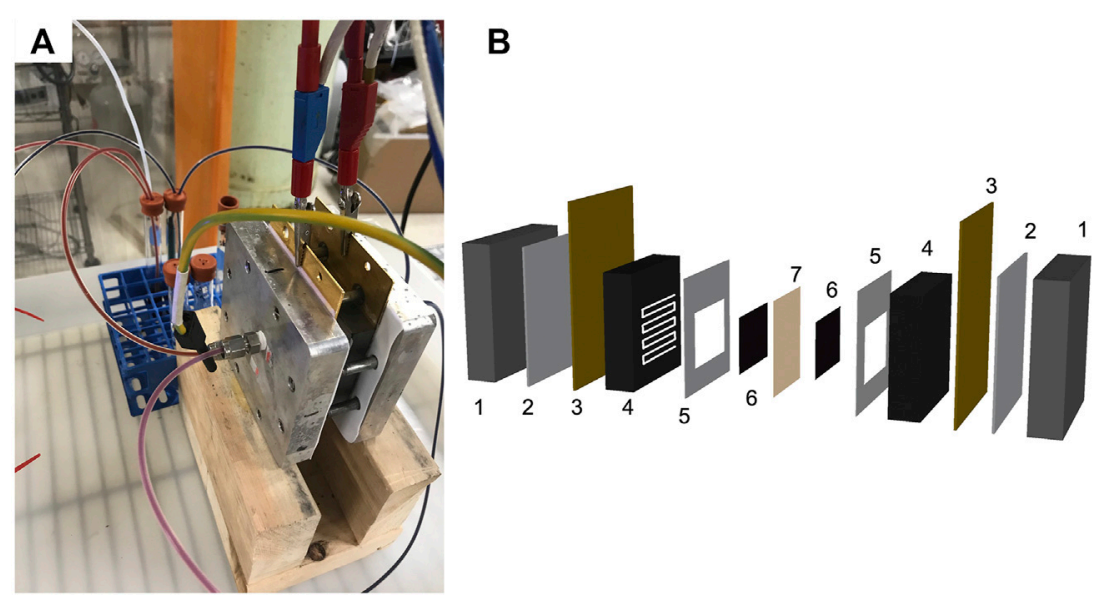

FIGURE 3 | Redox flow battery (A) photo and (B) exploded diagram of the cell. The cell components are (1) metal plates, (2) polytetrafluoroethylene (PTFE) insulating layers, (3) gold coated current collectors, (4) poco graphite bipolar plates with serpentine flow fields, (5) PTFE gaskets, (6) porous carbon electrodes, and (7) a membrane.

\section{Cell Design and Instrumentation}

The RFB (Figure 3) consisted of a two-electrode adapted fuel cell hardware design (Aaron et al., 2012), porous carbon electrodes, as received (SGL carbon felt GFD $2.5 \mathrm{~mm}$ or SGL Sigracell carbon fiber $350 \mu \mathrm{m}$ ), a Fumatech F-930-P cation exchange membrane, and a MasterFlex peristaltic pump. The battery was operated using a Biologic VSP potentiostat with VMP3B-20 (20 amp) booster controlled by EC-lab software.

\section{Polarization Curve Analysis}

The RFB was initially charged using chronoamperometry (CA) with an applied voltage of $1.4 \mathrm{~V}$, to $\sim 50 \%$ state of charge (SOC). A single-pass flow design was then used for polarization curve analysis to ensure a constant state of charge for electrolytes entering the cell. Due to the large volumes of electrolytes required for single-pass experiments, a relatively slow flow rate of $\sim 2.5 \mathrm{ml} \mathrm{min}^{-1}$ was employed for feasibility. Chronoamperometry was used to determine the steady state current at successive overpotentials starting from the open circuit voltage and incrementing in $25-50 \mathrm{mV}$ steps. The potential was applied for a long enough time to reach the steady state current at each step; $2 \mathrm{~min}$ in this study. Electrolyte flow generated by the peristaltic pump caused oscillations in the measured current, and therefore, the current was averaged over a few oscillations at the end of each 2 min potential step to obtain a value for the steady state current. Additionally, PEIS was used to determine the resistance through the cell, following the $2 \mathrm{~min}$ potential step. This was performed by superimposing a sinusoidal potential to the existing applied potential for each step. This perturbation had a magnitude of $5-10 \mathrm{mV}$ and a frequency of $10-1 \mathrm{kHz}$. The areal specific resistance was calculated from the product of the cell resistance (obtained from the HFR) and the geometric area of the electrode as shown in the following equation:

$$
\operatorname{ASR}\left(\Omega \cdot \mathrm{cm}^{2}\right)=\mathrm{HFR} \cdot \text { area }
$$

\section{Preliminary Cell and Electrolyte Optimization}

Preliminary cell optimization was performed by modifying the cell architecture (electrode compression) and components (electrodes) and electrolyte compositions. Following modification, the maximum steady state current and areal specific resistance were recorded. Charging was performed by chronoamperometry, as previously described. However, instead of recording the entire polarization curve, only the steady state current and resistance corresponding to a potential step with the largest magnitude overpotential were recorded $\left(\mathrm{E}_{\text {applied }}=0.1 \mathrm{~V}\right)$.

\section{Cycling}

A recirculating flow design using a flow rate of $10 \mathrm{ml} \mathrm{min}^{-1}$, with $350 \mu \mathrm{m}$ SGL carbon fiber electrodes, and 30\% expansion on the electrodes were used for cycling experiments. The posolyte and negolyte were ferrocene- and menadione-SDS microemulsions with a composition of $8.75 \%$ SDS 8.75\% 1-butanol, 52.5\% $0.5 \mathrm{~m} \mathrm{KNO}_{3}$ (aq), and $30 \%$ redox active species/toluene solution, by mass $\left(4.8 \mathrm{Ah} \cdot \mathrm{L}^{-1}\right)$. Galvanostatic cycling was performed at $5 \mathrm{~mA} \mathrm{~cm}^{-2}$, for four cycles.

\section{Metrics Calculations}

Coulombic efficiency $\left(\eta_{C}\right)$, voltage efficiency $\left(\eta_{U}\right)$, and energy efficiency $\left(\eta_{E}\right)$ were calculated from cycling data using the following equations:

$$
\begin{gathered}
\eta_{C}=\frac{Q_{D}}{Q_{C}} \\
\eta_{U}=\frac{U_{D}}{U_{C}} \\
\eta_{E}=\eta_{C} \cdot \eta_{U}
\end{gathered}
$$

where $\mathrm{Q}$ is charge, $\mathrm{U}$ is voltage, and the subscripts $\mathrm{D}$ and $\mathrm{C}$ define discharging and charging steps, respectively. 


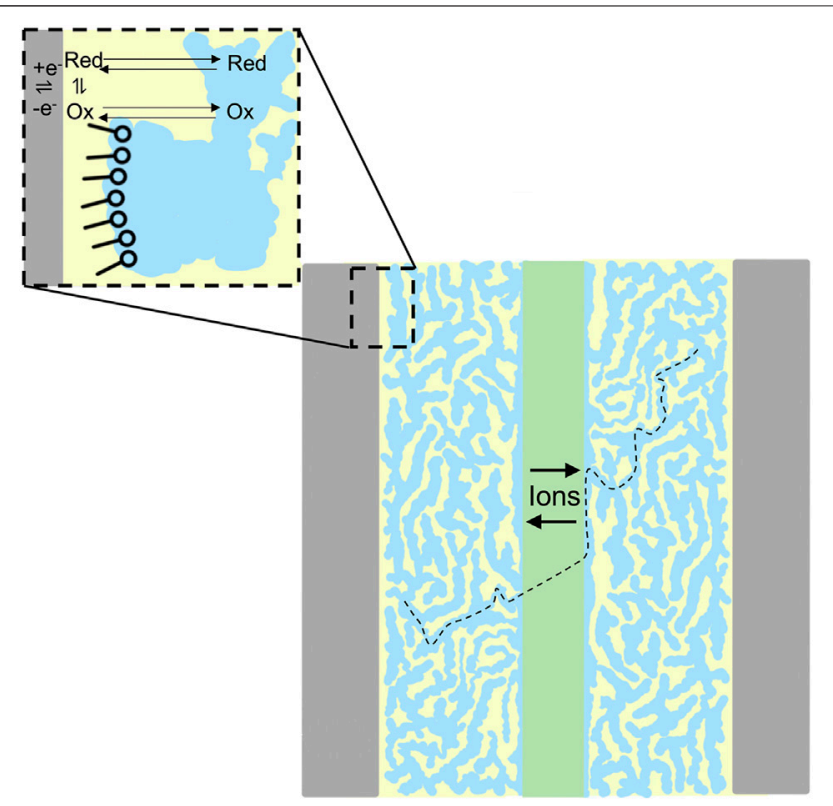

SCHEME 1 | An illustration of a redox flow battery with bicontinuous microemulsion electrolytes comprised of water (blue) and oil (yellow) phases separated by surfactant (inset). lon conduction (black dashed line) is depicted through the aqueous phase and membrane. Redox reactions are shown in the oil phase (inset).

\section{RESULTS AND DISCUSSION}

As we previously described (Aaron et al., 2011), polarization curve analysis readily reveals the mechanisms responsible for inefficiencies. Polarization curves provide a correlation between RFB efficiency and the physical behavior (electron transfer rates, mass transport, through-plane membrane resistivity) of electrolytes. Therefore, this analysis can diagnose properties of novel electrolytes that must be altered to optimize performance. We show in Scheme 1 an illustration of a novel microemulsion RFB indicating simultaneous ion conduction through the aqueous phase and redox reactions in the oil phase. The performance of a polysorbate 20 microemulsion $\mathrm{RFB}$ at constant $\sim 50 \%$ state of charge, with a $4.9 \mathrm{mM}$ ferrocene posolyte and $2.5 \mathrm{mM}$ menadione negolyte was evaluated using polarization curve analysis, as shown in Figure 4. The RFB used for that experiment consisted of a zero-gap cell (Aaron et al., 2012) Fumasep F-930 cation exchange membrane $(\sim 30 \mu \mathrm{m}$ thickness) with SGL GFD carbon felt electrodes $\left(5 \mathrm{~cm}^{2}\right.$ area, $2.5 \mathrm{~mm}$ thickness, 99\% compression) where electrolytes passed through the cell at a flow rate of $2.4 \mathrm{ml} \cdot \mathrm{min}^{-1}$. There is minimal loss in the kinetic polarization region, indicating facile electron transfer. Ohmic polarization is quantified using the areal specific resistance (ASR), the product of the high frequency resistance and the geometric electrode area. Throughout the porous electrode, ionic and electronic paths exist in parallel, with the electronic path having far less resistance. The ASR is then the sum of electrical resistances in the cell components and the ionic resistance through the membrane which separates the

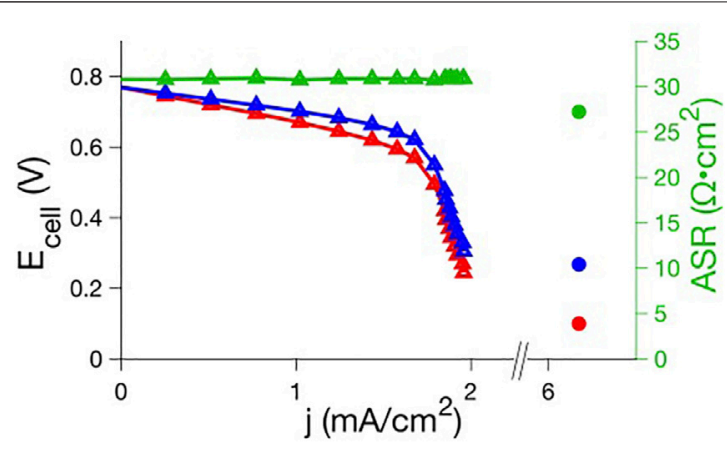

FIGURE 4 | Raw (red) and iR-compensated (blue) polarization curve and ASR (green) data for ferrocene posolyte-menadione negolyte polysorbate 20 microemulsion RFBs. A full polarization curve for a preliminary system (triangles; 89.9\% $0.5 \mathrm{~m} \mathrm{KNO}_{3}$ aq, $7.5 \%$ polysorbate $20,1.6 \% 1$ -

butanol, 1.0\% toluene; carbon felt electrodes with 99\% compression; $4.9 \mathrm{mM}$ ferrocene, $2.5 \mathrm{mM}$ menadione) is compared to the maximum current density and ASR for an optimized system (circles; 20\% $1.5 \mathrm{~m} \mathrm{KNO}_{3}$ aq, 47\% polysorbate 20, 10\% 1-butanol, 23\% toluene; carbon fiber electrodes with $30 \%$ expansion; $160 \mathrm{mM}$ ferrocene, $80 \mathrm{mM}$ menadione)).

electrodes. Electrical resistances are significantly smaller than ionic resistance through the membrane, and therefore, the ASR is mostly attributed to ionic resistance through the membrane. That is, for an ASR measurement, the electrode behaves as an electronic short circumventing the ionic pathway and the ASR does not probe the resistance of the electrolyte within the electrode. The ASR was $\sim 30 \Omega \mathrm{cm}^{2}$, one to two orders of magnitude larger than the ASR reported for vanadium redox flow batteries (Aaron et al., 2011), indicating significant ionic resistance through the membrane for polysorbate 20 microemulsions.

Following iR-compensation, the polarization curve in the linear region still exhibits a significant negative slope which suggests effects related to mass transport limitations inside the porous electrodes. In this region of the polarization curve, losses associated with transport of ions and redox species through the porous electrode, i.e. distributed resistance effects, dominate (Aaron et al., 2011). To access layers deeper within the electrode, one accrues more and more ionic resistance to bring ions (or charged reactants) in or out of the electrode. Put somewhat differently, functionally, in the polarization experiment, one is forced to bring ions/reagents in or out through the ionic conduction pathway in the porous electrode and thus, after iR correction, this looks like an additional iR loss (which we also refer to as a 'pseudo-IR) since the polarization curve is linear. This is an inherent transport loss that is distinct from that leading to a limiting current.

This loss is further supported by previous studies that have shown the diffusion of ferrocene in this microemulsion system is relatively slow $\left(\sim 10^{-7} \mathrm{~cm}^{2} \mathrm{~s}^{-1}\right)$ based on electrochemical measurements (Peng et al., 2020). There is also a clear mass transport polarization region associated with reactant starvation causing a maximum current density of $\sim 2 \mathrm{~mA} \cdot \mathrm{cm}^{-2}$ (Figure 4). In the absence of this limiting current, the linearly projected current density would be approximately $10 \mathrm{~mA} \cdot \mathrm{cm}^{-2}$. Total mass 
transport polarization likely originates from (1) over-compressed electrodes, (2) slow flow rate, (3) low concentration of active material, and/or (4) slow diffusion through the porous electrode.

In an open system such as a flow battery, an important criterion for practical success is a maximized current density. Even with mass transport limitations, normalizing current density to concentration gives $\sim 400 \mathrm{~mA} \cdot \mathrm{cm}^{-2} \mathrm{M}^{-1}\left(\mathrm{j}_{\max } \approx\right.$ $2 \mathrm{~mA} \cdot \mathrm{cm}^{-2}$ for a $5 \mathrm{mM}$ ferrocene solution). In comparison, early vanadium redox flow battery polarization studies reported $\sim 100 \mathrm{~mA} \cdot \mathrm{cm}^{-2} \mathrm{M}^{-1}$ limiting current densities for a similar $\left(2.0 \mathrm{ml} \cdot \mathrm{min}^{-1}\right)$ flow rate (Aaron et al., 2011). On a per molar basis, this appears promising. However, application requires larger total current densities which can be optimized through cell (electrode type, electrode compression) and electrolyte (chemistry and concentrations of salt, oil, surfactant, cosurfactant, redox species) engineering. Preliminary cell optimization was performed using polysorbate-based microemulsions. Electrode type, electrode compression, and microemulsion composition were varied in different combinations and the maximum current density was recorded. The best performance obtained from polysorbate 20 microemulsion electrolytes was a maximum current density of $\sim 6 \mathrm{~mA} \cdot \mathrm{cm}^{-2}$ for a similar state of charge and flow rate using a $160 \mathrm{mM}$ ferrocene posolyte and an $80 \mathrm{mM}$ menadione negolyte. Better performance was achieved through cell and electrolyte modifications by replacing carbon felt electrodes with thinner carbon fiber electrodes (SGL carbon fiber electrode with $5 \mathrm{~cm}^{2}$ area and $350 \mu \mathrm{m}$ thickness), decreasing the electrode compression (-30\% "compression") and changing redox species concentration and microemulsion composition. Despite the changes, the maximum current increased by only a factor of $\sim 3$ (Figure 4). In the absence of a full polarization curve, it is unclear how ionic or mass transport resistances contribute to the total loss. However, it is unlikely this polysorbate 20 electrolyte will be of practical use given the low current density and the large amount of surfactant needed to form a microemulsion, which limits energy density as well. Surfactant and cosurfactant are used only to maintain a stable microemulsion, and highly efficient surfactant/cosurfactant combinations should be used. Surfactant efficiency refers to the amount of oil and water that can be cosolubilized per amount of surfactant and is a common metric when evaluating (and optimizing) surfactants for microemulsion formulation (Salager et al., 2005). Polysorbate 20/1-butanol has poor efficiency with respect to toluene in this system: $37 \%$ polysorbate 20 and $8 \%$ 1-butanol is required to reach $30 \%$ toluene solutions. This microemulsion was chosen as an initial system based on our previous characterization of the phase behavior and corresponding electrochemistry. There is a complex relationship between microemulsion composition, nanometer domain structure, viscosity, conductivity, diffusion, and electrochemical behavior including redox potential and electron transfer kinetics (Mackay et al., 1990; Mackay et al.,1996; Peng et al., 2020; Shen et al., 2021). Therefore, prior knowledge of composition-property relationships could aid in analysis for electrolytes that had not yet been tested in RFBs. The polysorbate 20 system used in this study is bicontinuous (Peng et al., 2020), having both connected oil and water domains. Bicontinuous microemulsions are desirable because oil concentration is maximized while maintaining connected aqueous domains, which are needed for a conductive solution. Additionally, diffusion of oil-soluble redox active species will be greater than in oil droplet systems, increasing current density. However, polarization curve data shows that even when redox species concentration is near maximal for this system, the current density is only $\sim 6 \mathrm{~mA} \mathrm{~cm}^{-2}$ (under the given experimental conditions). Due to the limitations of the polysorbate system, a microemulsion with a more efficient surfactant (SDS) was then tested, to determine the effect of changing surfactant on polarization curve behavior and current density. Polarization analysis with SDS microemulsions was performed using the cell architecture (electrode type and compression) for which the polysorbate microemulsion system exhibited maximum current density.

SDS is a well-characterized surfactant (Chevalier and Zemb 1990; Ben-Shaul and Gelbart 1994; Acosta et al., 2003; Acosta et al. 2008) that has been used in electrochemical studies (Georges et al., 1987; Abbott et al., 1992; Mackay et al., 1996). Microemulsions up to $30 \%$ toluene can be formed using only 8.75\% SDS, $8.75 \%$ 1-butanol, and $52.5 \% 0.5 \mathrm{~m} \mathrm{KNO}_{3}$ aq., by mass. These SDS microemulsions were verified to be bicontinuous using proton diffusion NMR (Figure 5A). The decays in peak areas corresponding to protons from water and toluene were fit to decaying exponential functions (Figure 5B) to obtain diffusivities (Table 1). Errors in the fits (Table 1) were several orders of magnitude smaller than the determined diffusivities, which are reasonable in magnitude. The diffusivities of toluene and water are on the same order of magnitude and therefore the microemulsion is considered to be bicontinuous (Lindman et al., 1999). The idea behind this conclusion is that for a non-continuous oil or water phase, NMR diffusion measurements will report orders of magnitude differences since the non-continuous phase. Diffusion NMR spectra for SDS microemulsion solubilized ferrocene (Figure 5C) and menadione (Figure 5E) exhibited similar signal decays (Figures 5D,F, respectively) and diffusivities (Table 1) to the blank microemulsion. The small changes in water and toluene diffusivities between blank and redox solubilized systems may result from minor changes in interfacial curvature of the surfactant layer or from solvation effects (Lindman et al.,1999).

The effect of surfactant choice on electrochemical behavior of ferrocene and menadione was qualitatively analyzed using cyclic voltammetry $(\mathrm{CV})$. Electron transfer kinetics are more facile for both menadione (Figure 6A) and ferrocene (Figure 6B) in SDS microemulsions compared to polysorbate 20 microemulsions as indicated by the shape of the cyclic voltammogram. Additionally, the peak current density for ferrocene is approximately three times larger for the SDS microemulsion than the polysorbate 20 microemulsion. This behavior may be explained by both faster kinetics and increased diffusion. Menadione electron transfer in a polysorbate 20 microemulsion (at $\sim 30 \%$ toluene) appears irreversible, but in an SDS microemulsion the voltammogram exhibits both oxidation and reduction peaks. While the effect of microemulsion component ratios on electrochemical behavior 


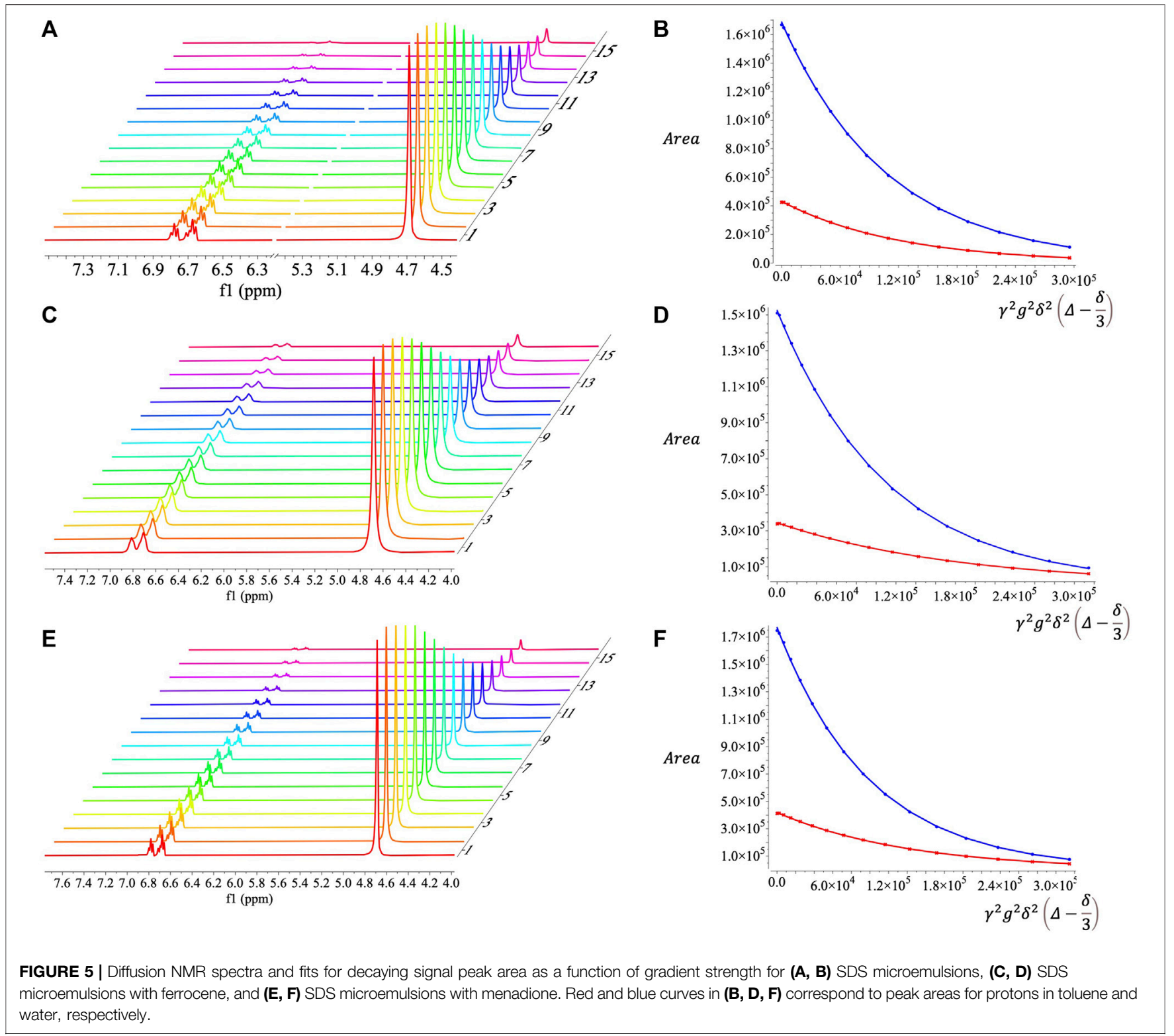

TABLE 1 | NMR diffusivities for SDS microemulsions.

\begin{tabular}{|c|c|c|c|c|}
\hline Microemulsion & Toluene $\left(\times 10^{-6} \mathrm{~cm}^{2} \mathrm{~s}^{-1}\right)$ & Toluene fitting error $\left(\times 10^{6}\right)$ & Water $\left(\times 10^{-6} \mathrm{~cm}^{2} \mathrm{~s}^{-1}\right)$ & Water fitting error $\left(\times 1^{6}\right.$ \\
\hline Ferrocene & 5.509 & 0.02693 & 8.996 & 0.02344 \\
\hline
\end{tabular}

was previously investigated (Peng et al., 2020), these findings emphasize the importance of surfactant chemistry.

How does RFB performance using SDS microemulsions compare to polysorbate 20 microemulsions? A polarization curve for a ferrocene $(192 \mathrm{mM})$ - menadione $(89 \mathrm{mM})$ SDS microemulsion (28\% toluene, 9\% SDS, 9\% 1-butanol, 54\% $\mathrm{KNO}_{3}$ aq., by mass) RFB is shown in Figure 7. Similar to the polysorbate 20 system, mass transport loss both in the form of diffusive transport loss (linear region) and concentration polarization loss (limiting current) is observed. Again, we note that while both effects have an origin in mass transport, they are somewhat distinct. Linear mass transport loss originates from transport of ions through the porous electrode, as described above, while the loss at greater current densities originates 

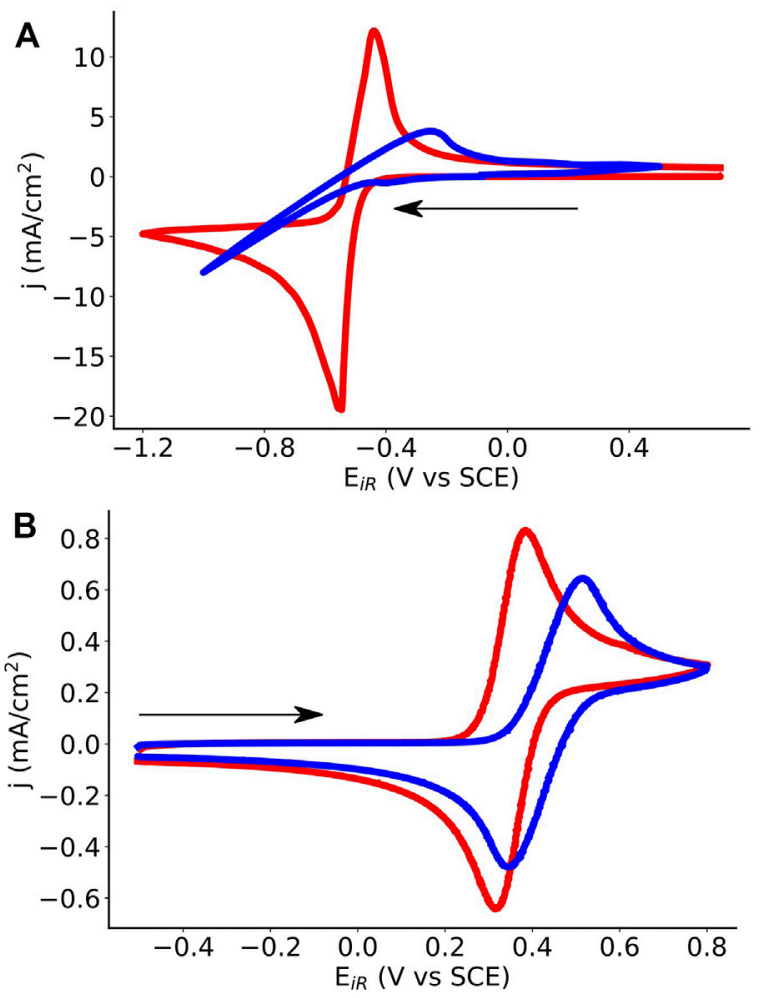

FIGURE 6 | Cyclic voltammograms comparing SDS (red) and polysorbate 20 (blue) microemulsions for (A) menadione negolyte and (B) ferrocene posolyte solutions. SDS microemulsions are 54\% $0.5 \mathrm{~m} \mathrm{KNO}_{3}$ (aq), 28\% toluene, 9\% SDS, and 9\% 1-butanol, by mass. Polysorbate microemulsions are $22 \% 2 \mathrm{~m} \mathrm{KNO}_{3}$ (aq), 31\% toluene, 39\% polysorbate 20 , and $8 \% 1$-butanol, by mass. Menadione concentrations are $0.13 \mathrm{M}$ in SDS and $0.3 \mathrm{M}$ in polysorbate microemulsions. Ferrocene concentrations are $\sim 5 \mathrm{mM}$ in SDS and polysorbate microemulsions. Voltammograms were recorded using glassy carbon working electrodes, saturated calomel reference electrodes, and Pt wire counter electrodes using a scan rate of $50 \mathrm{mV} \cdot \mathrm{s}^{-1}$. Sweep direction is indicated by the arrow.

from reagent starvation, which is essentially determined by the overall solution flow rate into the cell, the concentration of reagents and intrinsic permeability of the electrode. The presence of these losses is due, in part, to experimental design. The polarization curves were performed using single-pass flow to ensure a constant state of charge during measurement. Due to the single-pass experimental design, a slow flow rate $\left(2-3 \mathrm{ml} \cdot \mathrm{min}^{-1}\right)$ was used to reduce the amount of sample required. As flow rate slows, there is likely to be increased mass transport loss throughout the polarization curve (Sun et al., 2014). However, there is a greater pseudoiR loss, loss originating from ion coupled-redox species mass transport in the porous electrodes, in the polysorbate RFB than the SDS RFB, as demonstrated by the slopes of the iRcompensated polarization curves in the linear region. The SDS iR-compensated polarization curve exhibits a slope that is approximately an order of magnitude smaller than that of the polysorbate system. While this may be as dependent on the carbon electrode as the microemulsion, the exact nature of

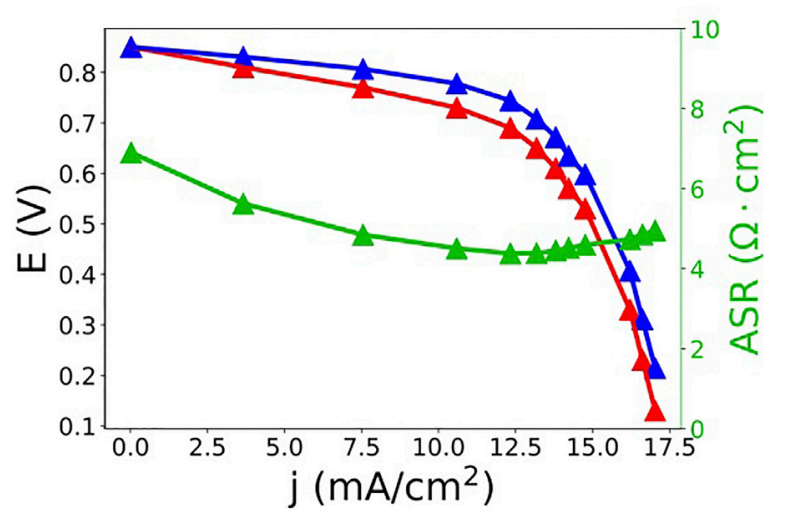

FIGURE 7|Raw (red) and iR-compensated (blue) polarization curve and ASR (green) data for a ferrocene (192 mM) - menadione $(89 \mathrm{mM})$ SDS microemulsion (28\% toluene, $9 \%$ SDS, $9 \%$ 1-butanol, $54 \% \mathrm{KNO}_{3}$ aq., by mass) RFB.

microemulsion-porous carbon electrode interaction has not been thoroughly investigated yet.

Table 2 compares the maximum current densities, ASRs, and energy densities between polysorbate 20 and SDS microemulsion electrolytes used in an RFB with similar concentrations of ferrocene $(\sim 0.16 \mathrm{M}$ for the polysorbate 20 microemulsions and $\sim 0.19 \mathrm{M}$ for the SDS microemulsions) and menadione $(\sim 0.08 \mathrm{M}$ for the polysorbate 20 microemulsions and $\sim 0.09 \mathrm{M}$ for the SDS microemulsions). Maximum current density reached $17.5 \mathrm{~mA} \mathrm{~cm}^{-2}$, three times the mass transport limited current density observed for the polysorbate 20 system using similar redox species concentrations. ASR decreased from 27 to $\sim 5 \Omega \mathrm{cm}^{2}$ when polysorbate was replaced with SDS. The decrease in ASR results from increased ionic transport. Surprisingly, the conductivity of the SDS system (28/9/9/54 toluene/SDS/1-butanol $/ 0.5 \mathrm{~m} \mathrm{KNO}_{3}$, by mass) was $14 \mathrm{mS} \cdot \mathrm{cm}^{-1}$ compared to $21 \mathrm{mS} \cdot \mathrm{cm}^{-1}$ for the polysorbate system $(1 / 7.5 / 1.6 /$ 89.9 toluene/polysorbate 20/1-butanol/0.5 $\mathrm{m} \mathrm{KNO}_{3}$, by mass). However, ASR accounts for membrane resistance and it is currently unclear how different microemulsions interact with porous carbon electrodes and membranes to influence ionic transport. While it is beyond the scope of this work, a thorough understanding of these interactions is needed if microemulsion RFBs are to be optimized.

The observed decrease in resistance most likely contributed to the increase in current density as mass transport losses in the electrode lead to uncompensated losses at mid-range current densities.

Volumetric capacities and energy densities are similar for both systems because the total amount of toluene solubilized, and therefore total concentration of redox active material was nearly equivalent. A theoretical "optimized" microemulsion electrolyte can be hypothesized from literature values and results from previously mentioned polarization curve studies. Using Ohm's law and the ASR from the SDS polarization curve experiment $\left(5 \Omega \mathrm{cm}^{2}\right)$, a theoretical current density can be determined. For a bicontinuous microemulsion with an extended potential window 
TABLE 2 | Microemulsion RFB-best performance comparison.

\begin{tabular}{|c|c|c|c|c|}
\hline Microemulsion & $\operatorname{Max} \mathrm{j}\left(\mathrm{mA} \cdot \mathrm{cm}^{-2}\right)$ & ASR $\left(\Omega \cdot \mathrm{cm}^{2}\right)$ & $\mathrm{C}\left(\mathrm{Ah} \cdot \mathrm{L}^{-1}\right)$ & $E\left(W h \cdot L^{-1}\right)$ \\
\hline Polysorbate 20 & 6.2 & 27 & 4.29 & 0.5 \\
\hline SDS & 17.5 & $\sim 5$ & 4.82 & 0.5 \\
\hline Theoretical best & *17,700 & ${ }^{\star} 0.13$ & 54 & 124 \\
\hline
\end{tabular}

of $\sim 2.3 \mathrm{~V}$ (Iwunze et al., 1990), the maximum current density would be $460 \mathrm{~mA} \cdot \mathrm{cm}^{-2}$. However, this is not an optimized ASR value. Our lab has previously demonstrated that current densities in VRFB could be increased to $5 \mathrm{~A} / \mathrm{cm}^{2}$, in part by reducing the ASR to only $0.13 \Omega \mathrm{cm}^{2}$ (Elgammal et al., 2017). This is a dramatic improvement over early VRFBs which reported ASR values of $\sim 4 \Omega \mathrm{cm}^{2}$ (Sun and Skyllas-Kazacos 1992). If the ASR in the microemulsion RFB shown here was reduced to $0.13 \Omega \mathrm{cm}^{2}$, the theoretical best current density, in the absence of other voltage losses (polarization, pseudo-iR, and mass-transport), would be $17.7 \mathrm{~A} / \mathrm{cm}^{2}$, due to the large potential window. Obviously, this extrapolation likely dramatically overstates the achievable increase since other factors besides ASR will undoubtedly dominate the losses as ASR is improved (see the final discussion below). However, decreasing the ASR can have a major effect.

If a nonpolar redox species that undergoes two electron transfer reactions (e.g., quinones) was dissolved in this microemulsion at a concentration of $1 \mathrm{M}$ overall, the theoretical volumetric capacity $54 \mathrm{Ah} \cdot \mathrm{L}^{-1}$. If the cell voltage was at the limit of the potential window $(2.3 \mathrm{~V})$, the energy density would be $124 \mathrm{Wh} \cdot \mathrm{L}^{-1}$. Although, the SDS and polysorbate systems do not yet approach the theoretical best, they provide a proof of concept which can be improved through future optimization, as previously shown for VRBs in our lab (Elgammal et al., 2017). Furthermore, this is more directly comparable to a non-aqueous redox flow battery (NARFB) and our nonoptimized current density without any normalization is already on the same order of magnitude of many NARFBs. Achieving high current density, while not the ONLY limitation of non-aqueous systems, is critical to keeping cell counts and concomitant materials costs low and, indeed, was the primary way in which RFBs advanced in the past decade. In fact, because of the low conductivity of non-aqueous media, current density is quite limited in NARFBs and that is a major weakness of such systems.

SDS microemulsions show improved performance over polysorbate 20 microemulsions, but how stable are these electrolytes? To test the stability of the system, galvanostatic cycling was performed at $5 \mathrm{~mA} \mathrm{~cm}{ }^{-2}$. Charge-discharge cycles are shown in Figure 8, and the derived efficiency metrics (Coulombic, voltage, and energy) and capacities per cycle were calculated are presented in Table 3. Over four cycles, Coulombic efficiency $\left(\eta_{C}\right)$ was relatively constant $(94-95 \%)$, but less than 99\%, indicating possible crossover or side reactions (Winsberg et al., 2017). Additionally, charge and discharge capacity decreased by at least $35 \%$, another indication of crossover or coupled homogeneous reactions consuming redox active material. Following cycling
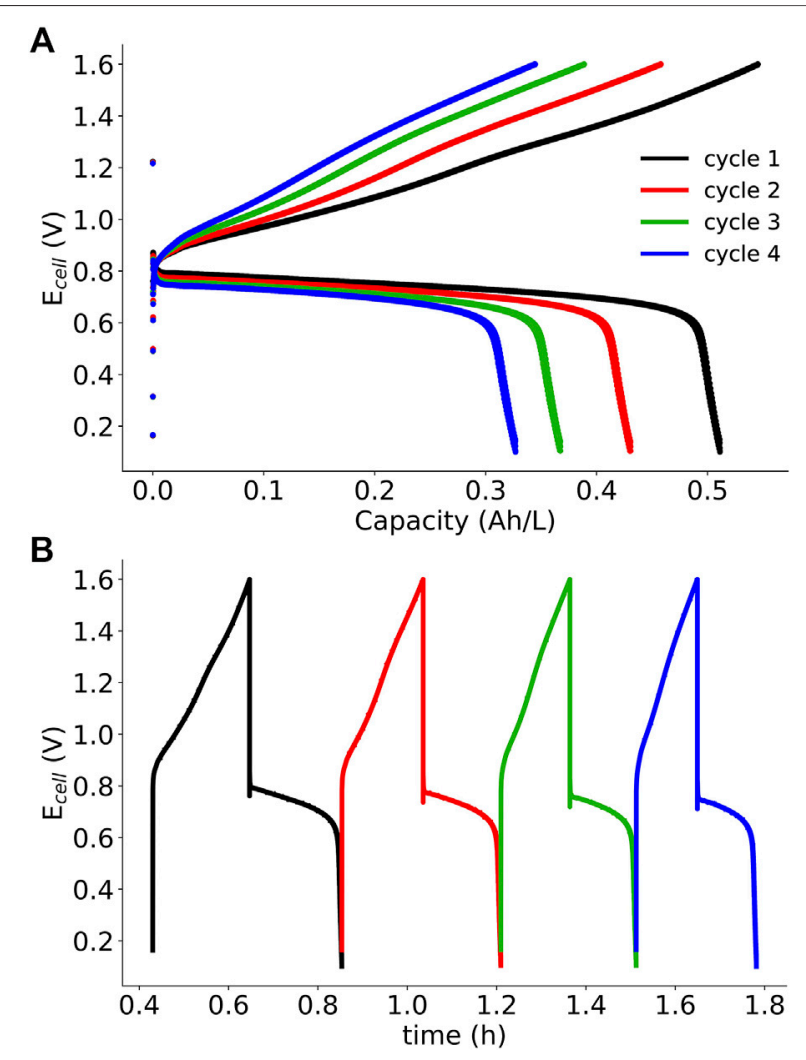

FIGURE 8 | SDS microemulsion RFB galvanostatic cycling data ( $5 \mathrm{~mA} \cdot \mathrm{cm}^{-2}$ ) for four cycles: (A) $E_{\text {cell }}$ vs capacity and (B) $E_{\text {cell }}$ vs time. SDS microemulsions are $8.75 \%$ SDS 8.75\% 1-butanol, 52.5\% $0.5 \mathrm{~m} \mathrm{KNO}_{3}$ (aq), and $30 \%$ redox active species/toluene solution. Ferrocene (192 mM) and menadione ( $89 \mathrm{mM}$ ) were the posolyte and negolyte redox active species, respectively.

experiments, the membrane was removed and examined. Material from the electrolyte had been taken up into the membrane, which is another indication of possible crossover. Microemulsion-membrane interactions are complex due to the presence of polar and non-polar components, and these interactions will be further investigated in a future work. It is possible that coupled homogeneous reactions involving menadione are responsible for capacity fade given the limited stability of most quinones (Kwabi et al., 2018). In an aprotic solvent, it is expected that menadione would be reduced to a dianion, which may be reactive. Additionally, microemulsions have been frequently used as microreactors to catalyze reactions by confining reactants to smaller domains (Rusling 1994). The 
TABLE 3 | Microemulsion RFB metrics.

\begin{tabular}{lccccc}
\hline Cycle & $\boldsymbol{\eta}_{\mathbf{C}}(\mathbf{\%})$ & $\boldsymbol{\eta}_{\mathbf{U}}(\mathbf{\%})$ & $\boldsymbol{\eta}_{\mathbf{E}}(\mathbf{\%})$ & $\mathbf{Q}_{\mathbf{D}}(\mathbf{m A} \mathbf{h})$ & $\mathbf{Q}_{\mathbf{C}}(\mathbf{m A} \cdot \mathbf{h})$ \\
\hline 1 & 94 & 60 & 56 & 5.1 & 5.5 \\
2 & 94 & 58 & 54 & 4.3 & 4.6 \\
3 & 95 & 56 & 53 & 3.7 & 3.9 \\
4 & 95 & 55 & 52 & 3.3 & 3.4 \\
\hline
\end{tabular}

confinement of reactive quinones may amplify coupled homogeneous reaction rates leading to the observed capacity fade.

Voltage $\left(\eta_{U}\right)$ and energy $\left(\eta_{E}\right)$ efficiencies range from 55 to $60 \%$ and $52-56 \%$, respectively; values which are lower than early vanadium RFBs. Voltage losses are the result of the combined overpotentials: kinetic, ohmic, and mass-transport. Through polarization curve analysis, it was determined that masstransport losses through the porous electrodes and ohmic losses through the membrane were significant. Although cycling experiments were performed at a greater flow rate than polarization curve experiments $(\sim 10 \mathrm{ml} / \mathrm{min}$ compared to $\sim 2.5 \mathrm{ml} / \mathrm{min}$ ), mass transport limitations may still be responsible for the observed voltage inefficiencies.

While the demonstrated use of microemulsion based electrolytes provided here is a first step, there is a substantial hill to climb to make these electrolytes as useful as possible. Electrolyte design is motivated by the following objectives: (1) maximize energy density, (2) maximize current density, (3) minimize capacity fade, and (4) minimize cost. Each objective is dependent on microemulsion and redox species choice, as well as RFB cell design and operation parameters. For typical oil-water-surfactant-cosurfactant microemulsions, there are 15 independent variables (for a quaternary mixture, three of the four component fractions are independent, and one is constrained) that must be optimized to meet these objectives (Table 4). A closer look at the first objective, maximizing energy density, will illustrate the complexity of this problem. Energy density can be described mathematically as:

$$
E=C \cdot U=n F[\text { redox }] \cdot U
$$

where $\mathrm{E}$ is the energy density $(\mathrm{J} / \mathrm{L}), \mathrm{C}$ is the volumetric capacity (coulomb/L), $\mathrm{U}$ is the voltage $(\mathrm{V}), n$ is the mole of electrons transferred per mole of redox species, F is Faraday's constant (coulombs/mole electrons), and [redox] is concentration of redox species $(\mathrm{M})$. The simplest case for a microemulsion electrolyte is a nonpolar redox species which exhibits a single, reversible outersphere electron transfer (e.g., ferrocene), fixing $n$ at one. Microemulsion formulation can increase energy density by increasing redox concentration $([$ redox $]$ ) and/or shifting the redox potential of the half reaction (which will influence the cell voltage, $U$ ). Assuming that all surfactant and cosurfactant are at the amphiphilic interface, the redox concentration is determined by solubility in the oil phase. Therefore, it is logical to choose an oil that will maximize redox solubility and a microemulsion composition that will maximize the oil phase. However, for an RFB application, this is achieved under the constraints that the microemulsion is single-phase and conductive.

Not all compositions of an oil, water (or brine), surfactant, cosurfactant mixture will generate single-phase microemulsions; water- or oil-swollen micelles, lyotropic liquid crystals, and macroemulsions are all possible outcomes (Moulik and Paul 1998). If surfactant content is too low, macroemulsions are often formed. Macroemulsions are kinetically stable dispersions of droplets that are several orders of magnitude larger than microemulsion domains. Given sufficient time, macroemulsions will phase separate into a microemulsion layer with excess oil and/or water layers. Energy density will decrease if excess phases are formed which do not participate in electrochemical reactions, and inefficiency will increase if energy input is needed to prevent phase separation. On the contrary, if surfactant content is excessive, energy density will decrease. The only purpose of the surfactant is to reduce the interfacial tension between the oil and water phases, allowing microemulsions to form. Once a single-phase microemulsion forms, additional surfactant will (generally) increase solution volume and thus, reduce energy density. Furthermore, the microemulsion must be conductive for electrochemical applications, implying a continuous aqueous network. Microemulsion conductivity is strongly correlated to structure, and while large oil content favors increasing energy density, it also favors water-in-oil droplet structures which are insulating. Therefore, an upper limit on oil content exists for each system that must not be exceeded. Oil-in-water droplets and bicontinuous microemulsions both satisfy the conductivity requirement, but bicontinuous systems will maximize energy density while maintaining a continuous aqueous phase. This means, for each possible quintenary (redox species, oil, brine, surfactant, cosurfactant) microemulsion, the component- and composition-dependent phase behavior must be understood if

TABLE 4 | Optimization objectives and independent variables.

\begin{tabular}{|c|c|c|}
\hline Number of variables & $\begin{array}{c}\text { Objectives } \\
\text { (dependent Variables) }\end{array}$ & Independent variables \\
\hline 1 & energy density & redox species ${ }^{a}$ \\
\hline 2 & current density & salt/acid/base ${ }^{a}$ \\
\hline 3 & capacity fade & $\mathrm{oil}^{\mathrm{a}}$ \\
\hline 4 & cost & surfactant $^{a}$ \\
\hline 5 & & cosurfactant $^{\mathrm{a}}$ \\
\hline 6 & & temperature ${ }^{a}$ \\
\hline 7 & & {$[\text { redox }]^{a}$} \\
\hline 8 & & lonic strength/pH ${ }^{\mathrm{a}}$ \\
\hline 9 & & oil phase fraction ${ }^{\mathrm{a}}$ \\
\hline 10 & & aqueous phase fraction ${ }^{a}$ \\
\hline 11 & & surfactant fraction ${ }^{\mathrm{a}}$ \\
\hline 12 & & cosurfactant fraction ${ }^{\mathrm{a}}$ \\
\hline 13 & & flow rate $e^{b}$ \\
\hline 14 & & membrane $e^{b}$ \\
\hline 15 & & electrode $^{\mathrm{b}}$ \\
\hline 16 & & flow field geometry ${ }^{\mathrm{b}}$ \\
\hline
\end{tabular}

In an oil-water-surfactant-cosurfactant system, the fraction of one of the components will be constrained (dependent).

${ }^{a}$ Microemulsion variables.

${ }^{b} R F B$, variables. 
bicontinuous systems with minimal surfactant are to be screened. This is a non-trivial, time-consuming task.

Additionally, energy density can be increased through microemulsion formulation by modifying the cell voltage term. This is accomplished by (1) extending the aqueous potential window (Peng et al., 2020) to enable to use of previously inaccessible redox species or (2) shifting redox potentials to increase cell voltage (Mackay et al., 1996). The mechanisms for these behaviors are not yet clear.

The remaining objectives are just as complex and may include RFB cell variables, as well. For example, current density is a function of potential-dependent kinetics for electron transfer at a porous electrode while conductivity is maintained through a membrane and mass transport is a function of flow rate. The electrode- and membrane interactions with the microemulsion, as shown in this work, further complicate microemulsion electrochemistry. Thus, improving the performance, i.e. current density or power density, of an RFB based on microemulsions will entail control over interactions with the physical cell components (i.e. electrodes and membranes). There are significant questions related to the wetting of each of those components and how it is affected by the surfactants, for example. Mass transport and interfacial electron transfer within porous electrodes is a major point of emphasis for achieving high performance. Significant progress is needed to optimize microemulsion formulation, specifically to increase the attainable solubility of the redox active species (to realize energy densities equal to or high than those achieved in other systems), and to understand and control interactions of the various electrolyte constituents with cell components.

Though the matrix of optimizations does indeed appear to be complex, this is a space that our group has navigated before with major gains in performance. Improvements in membranes, electrodes and cell design all contributed to these gains. The use of microemulsions in electrochemical conversions for RFBs and beyond offers a unique variable for manipulating the reaction pathways possible.

\section{CONCLUSION}

In this work, we have demonstrated the use of microemulsion electrolytes as potential "breakthrough" electrolytes by showing their use in model microemulsion RFBs. Microemulsions can incorporate novel organic redox species which were previously incapable of being used in combination with aqueous systems or

\section{REFERENCES}

Aaron, D. S., Liu, Q., Tang, Z., Grim, G. M., Papandrew, A. B., Turhan, A., et al. (2012). Dramatic Performance Gains in Vanadium Redox Flow Batteries through Modified Cell Architecture. J. Power Sourc. 206, 450-453. doi:10. 1016/j.jpowsour.2011.12.026

Aaron, D., Tang, Z., Papandrew, A. B., and Zawodzinski, T. A. (2011). Polarization Curve Analysis of All-Vanadium Redox Flow Batteries. J. Appl. Electrochem. 41, 1175-1182. doi:10.1007/s10800-011-0335-7 suffered from poor performance in non-aqueous systems due to low solubility or poor conductivity. Microemulsion RFB performance was improved by changing electrolyte composition and by hardware modification. Maximum current densities for the optimized microemulsion redox flow battery are comparable to many NARFBs. However, performance was limited by inefficiencies and capacity fade. Voltage and energy efficiencies were less than $60 \%$ and most likely arise from mass transport overpotentials, as identified by polarization curve analysis. Coulombic inefficiency and Capacity fade may be related to crossover as well as coupled side reactions involving reactive menadione anions confined to small nonpolar domains. Similar to many quinone RFBs, overcoming capacity fade will lead to increased applicability of the microemulsion RFB. Based on this early work microemulsions have great potential as electrolytes if major efforts aimed at optimizing composition of the electrolytes and tailoring cell components for this application are undertaken. The unique combination of conductive aqueous and reactive oil phases may overcome limitations of existing aqueous and non-aqueous systems, furthering the promise of RFBs as energy storage devices.

\section{DATA AVAILABILITY STATEMENT}

The raw data supporting the conclusions of this article will be made available by the authors, without undue reservation.

\section{AUTHOR CONTRIBUTIONS}

The project and experimental design were developed by BB and TZ. Experiments were performed by BB; cyclic voltammetry experiments were performed with assistance from KN. NMR data analysis was performed by $\mathrm{BB}$ with assistance from AI. Conductivity cell was designed and built by GG. The manuscript was written by BB and TZ.

\section{FUNDING}

This work was supported as part of the Breakthrough Electrolytes for Energy Storage (BEES), an Energy Frontier Research Center funded by the United States. Department of Energy, Office of Science, Basic Energy Sciences under Award \# DE-SC0019409.

Abbott, A. P., MiawMiaw, C. L., and Rusling, J. F. (1992). Correlations between Solvent Polarity Scales and Electron Transfer Kinetics and an Application to Micellar media. J. Electroanalytical Chem. 327, 31-46. doi:10.1016/00220728(92)80134-p

Acosta, E. J., YuanYuan, J. S., and Bhakta, A. S. (2008). The Characteristic Curvature of Ionic Surfactants. J. Surfact Deterg 11, 145-158. doi:10.1007/ s11743-008-1065-7

Acosta, E., Szekeres, E., Sabatini, D. A., and Harwell, J. H. (2003). Net-Average Curvature Model for Solubilization and Supersolubilization in Surfactant Microemulsions. Langmuir 19, 186-195. doi:10.1021/la026168a 
Ben-Shaul, A., and Gelbart, W. M. (1994). "Statistical Thermodynamics of Amphiphile Self-Assembly: Structure and Phase Transitions in Micellar Solutions," in Micelles, Membranes, Microemulsions, and Monolayers. Editors W. M. Gelbart, A. Ben-Shaul, and D. Roux (New York: Springer). doi:10.1007/978-1-4613-8389-5_1

Chevalier, Y., and Zemb, T. (1990). The Structure of Micelles and Microemulsions. Rep. Prog. Phys. 53, 279-371. doi:10.1088/0034-4885/53/3/002

Elgammal, R. A., TangSun, Z. C., Sun, C.-N., Lawton, J., and Zawodzinski, T. A. (2017). Species Uptake and Mass Transport in Membranes for Vanadium Redox Flow Batteries. Electrochimica Acta 237, 1-11. doi:10.1016/j.electacta. 2017.03.131

Georges, J., Chen, J.-W., and Arnaud, N. (1987). Microemulsion Structure in the Lenticular Monophasic Areas of brine/SDS/pentanol/dodecane or Hexane Systems: Electrochemical and Fluorescent Studies. Colloid Polym. Sci 265, 45-51. doi:10.1007/bf01422663

Hou, Z., Zhang, X., Li, X., Zhu, Y., Liang, J., and Qian, Y. (2017). Surfactant Widens the Electrochemical Window of an Aqueous Electrolyte for Better Rechargeable Aqueous Sodium/zinc Battery. J. Mater. Chem. A. 5, 730-738. doi:10.1039/ c6ta08736a

Hu, B., DeBruler, C., Rhodes, Z., and Liu, T. L. (2017). Long-Cycling Aqueous Organic Redox Flow Battery (AORFB) toward Sustainable and Safe Energy Storage. J. Am. Chem. Soc. 139, 1207-1214. doi:10.1021/jacs.6b10984

Iwunze, M. O., Sucheta, A., and Rusling, J. F. (1990). Bicontinuous Microemulsions as media for Electrochemical Studies. Anal. Chem. 62, 644-649. doi:10.1021/ ac00205a021

Jha, B. K., Kulkarni, B. D., Vinod, M. P., and Vijayamohanan, K. (1995). An Unusual Electron-Transfer Behavior of Ferrocene in Aqueous Microemulsion Systems. Chem. Phys. Lett. 240, 442-448. doi:10.1016/0009-2614(95)00572-1

Kwabi, D. G., Lin, K., Ji, Y., GouletPollack, M.-A., De Porcellinis, D., Tabor, D. P., et al. (2018). Alkaline Quinone Flow Battery with Long Lifetime at $\mathrm{pH}$ 12. Joule, 2, 1894-1906. doi:10.1016/j.joule.2018.07.005

Lindman, Björn., Olsson, Ulf., and Söderman, Olle. (1999). "Characterization of Microemulsions by NMR," in Handbook of Microemulsion Science and Technology. Editors K. L. Mittal and P. Kumar (New York: Marcel Dekker).

Luo, J., Hu, B., Debruler, C., Bi, Y., Zhao, Y., Yuan, B., et al. (2019a). Unprecedented Capacity and Stability of Ammonium Ferrocyanide Catholyte in $\mathrm{pH}$ Neutral Aqueous Redox Flow Batteries. Joule 3, 149-163. doi:10.1016/j.joule.2018. 10.010

Luo, J., Hu, B., Hu, M., Zhao, Y., and Liu, T. L. (2019b). Status and Prospects of Organic Redox Flow Batteries toward Sustainable Energy Storage. ACS Energ. Lett. 4, 2220-2240. doi:10.1021/acsenergylett.9b01332

Mackay, R. A. (1994). Electrochemistry in Association Colloids. Colloids Surf. A: Physicochemical Eng. Aspects 82, 1-28. doi:10.1016/0927-7757(93)02610-q

Mackay, R. A., Myers, S. A., Bodalbhai, L., and Brajter-Toth, A. (1990). Microemulsion Structure and its Effect on Electrochemical Reactions. Anal. Chem. 62, 1084-1090. doi:10.1021/ac00209a023

Mackay, R. A., Myers, S. A., and Brajter-Toth, A. (1996). Interpreting Diffusion Coefficients of Electrochemical Probes in Oil-In-Water Microemulsions. Electroanalysis 8, 759-764. doi:10.1002/elan.1140080810

McCreery, R. L. (2008). Advanced Carbon Electrode Materials for Molecular Electrochemistry. Chem. Rev. 108, 2646-2687. doi:10.1021/cr068076m

Mo, C., and Li, X. (2007). Microstructure and Structural Transition in Coconut Oil Microemulsion Using Semidifferential Electroanalysis. J. Colloid Interf. Sci. 312, 355-362. doi:10.1016/j.jcis.2007.03.030

Moulik, S. P., and Paul, B. K. (1998). Structure, Dynamics and Transport Properties of Microemulsions. Adv. Colloid Interf. Sci. 78, 99-195. doi:10.1016/s00018686(98)00063-3

Oshawa, Y., Shimazaki, Y., and Aoyagui, S. (1980). The Half-Wave Potential and Homogeneous Electron-Transfer Rate Constant in Sodium Dodecyl Sulphate Micellar Solution. J. Electroanalytical Chem. Interfacial Electrochemistry 114, 235-246. doi:10.1016/s0022-0728(80)80450-5
Peng, J., Cantillo, N. M., Nelms, K. M., Roberts, L. S., Goenaga, G., Imel, A., et al. (2020). Electron Transfer in Microemulsion-Based Electrolytes. ACS Appl. Mater. Inter. 12, 40213-40219. doi:10.1021/acsami.0c07028

Peng, J., Cantillo, N. M., Xiao, Y., Nelms, K. M., Roberts, L. S., Goenaga, G., et al. (2021a). Decoupling Conductivity and Solubility in Electrolytes Using Microemulsions. J. Electrochem. Soc. 168, 080502. doi:10.1149/1945-7111/ac180b

Peng, J., Xiao, Y., Imel, A., Barth, B. A., Cantillo, N. M., Nelms, K. M., et al. (2021b). Electrolyte Effects on the Electrochemical Performance of Microemulsions. Electrochimica Acta 393, 139048. doi:10.1016/j.electacta.2021.139048

Qutubuddin, S. (1999). "Electrochemical Studies in Microemulsions," in Handbook of Microemulsion Science and Technology. Editors K. L. Mittal and P. Kumar (New York: Marcel Dekker).

Rusling, F. (1994). "Electrochemistry and Electrochemical Catalysis in Microemulsions," in Modern Aspects of Electrochemistry. Editors B. E. Conway, J. O'M. Bockris, and R. E. White (Springer US). doi:10.1007/ 978-1-4899-1733-1_2

Salager, J.-L., Antón, R. E., Sabatini, D. A., Harwell, J. H., Acosta, E. J., and Tolosa, L. I. (2005). Enhancing Solubilization in Microemulsions-State of the Art and Current Trends. J. Surfact Deterg 8, 3-21. doi:10.1007/s11743-005-0328-4

Shen, X., Sinclair, N., Wainright, J., Imel, A., Barth, B., Zawodzinski, T., et al. (2021). A Study of Ferrocene Diffusion in Toluene/Tween 20/1-Butanol/Water Microemulsions for Redox Flow Battery Applications. J. Electrochem. Soc. 168, 060539. doi:10.1149/1945-7111/ac0b26

Stejskal, E. O., and Tanner, J. E. (1965). Spin Diffusion Measurements: Spin Echoes in the Presence of a Time-Dependent Field Gradient. J. Chem. Phys. 42, 288-292. doi:10.1063/1.1695690

Sun, B., and Skyllas-Kazacos, M. (1992). Chemical Modification of Graphite Electrode Materials for Vanadium Redox Flow Battery Application-Part II. Acid Treatments. Electrochimica Acta 37, 2459-2465. doi:10.1016/00134686(92)87084-d

Sun, C.-N., Delnick, F. M., Aaron, D. S., Papandrew, A. B., Mench, M. M., and Zawodzinski, T. A. (2014). Resolving Losses at the Negative Electrode in AllVanadium Redox Flow Batteries Using Electrochemical Impedance Spectroscopy. J. Electrochem. Soc. 161, A981-A988. doi:10.1149/2.045406jes

Sun, C.-N., Mench, M. M., and Zawodzinski, T. A. (2017). High Performance Redox Flow Batteries: An Analysis of the Upper Performance Limits of Flow Batteries Using Non-aqueous Solvents. Electrochimica Acta 237, 199-206. doi:10.1016/j.electacta.2017.03.132

Winsberg, J., Hagemann, T., Janoschka, T., Hager, M. D., and Schubert, U. S. (2017). Redox-Flow Batteries: From Metals to Organic Redox-Active Materials. Angew. Chem. Int. Ed. 56, 686-711. doi:10.1002/anie.201604925

$\mathrm{Zu}$, X., and Rusling, J. F. (1997). Amphiphilic Ferrocene Alcohols as Electroactive Probes in Micellar Solutions and Microemulsions. Langmuir 13, 3693-3699. doi:10.1021/la962037w

Conflict of Interest: The authors declare that the research was conducted in the absence of any commercial or financial relationships that could be construed as a potential conflict of interest.

Publisher's Note: All claims expressed in this article are solely those of the authors and do not necessarily represent those of their affiliated organizations, or those of the publisher, the editors and the reviewers. Any product that may be evaluated in this article, or claim that may be made by its manufacturer, is not guaranteed or endorsed by the publisher.

Copyright $\odot 2022$ Barth, Imel, Nelms, Goenaga and Zawodzinski. This is an openaccess article distributed under the terms of the Creative Commons Attribution License (CC BY). The use, distribution or reproduction in other forums is permitted, provided the original author(s) and the copyright owner(s) are credited and that the original publication in this journal is cited, in accordance with accepted academic practice. No use, distribution or reproduction is permitted which does not comply with these terms. 